\title{
Photoluminescence Spectroscopy of CdTe/ZnTe Self-Assembled Quantum Dots
}

\author{
Nebojša Romčević, ${ }^{1}$ Maja Romčević, ${ }^{1}$ Radmila Kostić, ${ }^{1}$ Dušanka Stojanović, ${ }^{1}$ \\ Aleksandra Milutinović, ${ }^{1}$ Jelena Trajić, ${ }^{1}$ Grzegorz Karczewski, ${ }^{2}$ and Robert Galazka ${ }^{2}$ \\ ${ }^{1}$ Institute of Physics, 11080 Belgrade, Serbia \\ ${ }^{2}$ Institute of Physiscs, Polish Academy of Science, 02-668 Warsaw, Poland
}

Correspondence should be addressed to Nebojša Romčević, romcevi@phy.bg.ac.rs

Received 26 June 2009; Revised 27 October 2009; Accepted 9 November 2009

Recommended by Mohamed Sabry Abdel-Mottaleb

We present photoluminescence (PL) measurements of two different, 3 monolayers and 12 monolayers (ml), CdTe self-assembled quantum dot (SAQD) samples. The spectra were recorded in the temperature range $20 \mathrm{~K}-300 \mathrm{~K}$, with photoexcitation over the ZnTe barrier layer. PL spectra displayed two main emission bands. High-energy PL emission ( $\left.\mathrm{E}_{1}\right)$ is $\mathrm{ZnTe}$ LO like phonon$\left(\omega_{\mathrm{LO}}=204.2 \mathrm{~cm}^{-1}(3 \mathrm{ml}), \omega_{\mathrm{LO}}=207.3 \mathrm{~cm}^{-1}(12 \mathrm{ml})\right)$ assisted deexcitation. Dominant low-energy band $\left(\mathrm{E}_{2}\right)$ presents the direct deexcitation to ground state of the CdTe quantum dots.

Copyright ( 2009 Nebojša Romčević et al. This is an open access article distributed under the Creative Commons Attribution License, which permits unrestricted use, distribution, and reproduction in any medium, provided the original work is properly cited.

\section{Introduction}

Great interest has been observed recently in studying the properties of layers of semiconductor materials with a mismatch between their lattice parameters. Favourable conditions for the formation of self-assembled quantum dots are created in such layers [1]. The wide band-gap CdTe/ZnTe system is current interest because of its potential applications in short-wavelength optoelectronic devices, but the large lattice mismatch $(6.4 \%)$ makes it very difficult to grow $\mathrm{CdTe} / \mathrm{ZnTe}$ structure of high quality [2].

In the majority of experiments reported so far, dots with sizes larger than the exciton Bohr radius have been studied [3-5]. For instance, the typical size of II-VI CdSe QD's, where the exciton Bohr radius is equal to $3 \mathrm{~nm}$, ranges from slightly larger than this value [3] to even $15 \mathrm{~nm}$ in diameter [4]. In these cases then, leakage of the exciton wave function into the barriers is not expected to change significantly for QD size distributions within an ensemble. Consequently, for large QDs no size dependence of the exciton-LO phonon coupling is observed.

Detailed measurements of PL, photoluminescence excitation (PLE), and resonant PL spectroscopy on similar systems are performed in literature $[1,5]$. To study the carrier excitation processes in CdTe/ZnTe SAQD excitations below energy of $\mathrm{ZnTe}$ gap were used to identify major carrier excitation mechanisms in CdTe QD's [5].

In our earlier papers we using far-infrared spectroscopy [6], Raman spectoscopy [7], and resonant Raman spectroscopy [8] to investigate structural and phonon properties of CdTe/ZnTe SAQD. By extending this study to phtoluminescence spectroscopy we expect to obtain electronic structure and get complete picture of optical properties of these samples.

\section{Samples and Characterization}

Investigated samples were grown by molecular beam epitaxy on GaAs substrate. CdTe buffer layer, $4 \mu \mathrm{m}$ thick, was deposited on the substrate. After a $0.6 \mu \mathrm{m}$ thick $\mathrm{ZnTe}$ layer, 3 or 12 monolayers $(\mathrm{ml})$ of CdTe were deposited to form a random distribution of quantum dots. The dot layer was covered by $0.1 \mu \mathrm{m} \mathrm{ZnTe}$ capping layer, which is schematic presented in Figure 1. High mismatch of lattice parameters of CdTe (6.482 ̊́) and ZnTe (6.104 ̊́) provokes gathering of CdTe molecules and forming of quantum dots. Further details of the samples growth can be found in 


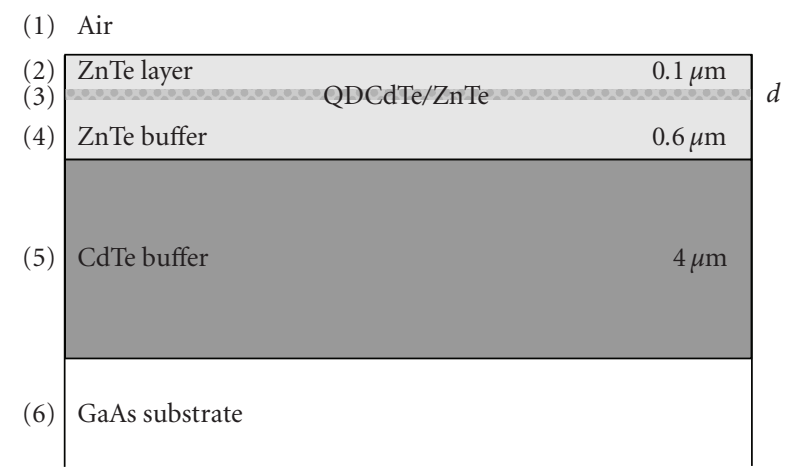

FIgURE 1: A schematic presentation of a six-layer structure.

[1]. It is important to note that the chosen sample set enables us to study quantum dots (QD) with different lateral sizes. Namely, CdTe QDs are very small, and, as estimated by transmission electron microscopy and magnetophotoluminescence measurements, their lateral size is of the order of $2-4 \mathrm{~nm}$ in diameter in sample with $3 \mathrm{ml}$. In second sample $(12 \mathrm{ml})$ there are no CdTe islands; that is, there is a layer with CdTe regions surrounded by alloyed material. This work is focussed to emphasize different properties of these two cases.

Photoluminescence spectra (PL) were excited by several lines of Ar-laser line ( $514.5 \mathrm{~nm}, 501.7 \mathrm{~nm}, 496.5 \mathrm{~nm}, 488 \mathrm{~nm}$ ) and $\mathrm{Kr}$-laser line $(647.5 \mathrm{~nm})$, measured using a Jobin Yvon model U-1000 monochromator, with a conventional photocounting system. All Ar-laser lines excite excitons over the ZnTe barrier layer.

\section{Results and Discussion}

The electronic band structure was characterized by a PL spectrum. PL spectra depend on energy excitation. In the case of argon laser excitations the PL spectra displayed two main emission peaks. Typical PL spectra are presented in Figures 2 and 3. Registered bands are separated and no additional analysis was needed to follow the temperature dependence of the energies corresponding to the maximum emission of these bands. Results of all measurements are presented in Figure 4.

We emphasize peaks positions for temperatures at the end of measurement interval. In the case of $3 \mathrm{ml}$; we have $2.26 \mathrm{eV}\left(E_{1}\right)$ and $1.84 \mathrm{eV}\left(E_{2}\right)$ at $T=300 \mathrm{~K}$, and $2.346 \mathrm{eV}$ $\left(E_{1}\right)$ and $2.17 \mathrm{eV}\left(E_{2}\right)$ at $T=20 \mathrm{~K}$. Change in $E_{1}$ energy is about $85 \mathrm{meV}$ and change in $E_{2}$ is about $330 \mathrm{meV}$. Redshift of the high-energy peak $E_{1}$ with increasing temperature is the same order of the shift that one would expect from the temperature-induced shrinking of the ZnTe band gap. This peak is registered even at room temperature. Red-shift of the low-energy peak $E_{2}$ with increasing temperature is much larger than the shift that one would expect from the temperature-induced shrinking of the CdTe band gap. In the case of $12 \mathrm{~mL}$; we have $2.307 \mathrm{eV}\left(E_{1}\right)$ and $2.11 \mathrm{eV}\left(\mathrm{E}_{2}\right)$ at $T=200 \mathrm{~K}$, and $2.337 \mathrm{eV}\left(E_{1}\right)$ and $2.13 \mathrm{eV}\left(\mathrm{E}_{2}\right)$ at $T=$ $20 \mathrm{~K}$. Change in $E_{1}$ energy is about $30 \mathrm{meV}$ and change in

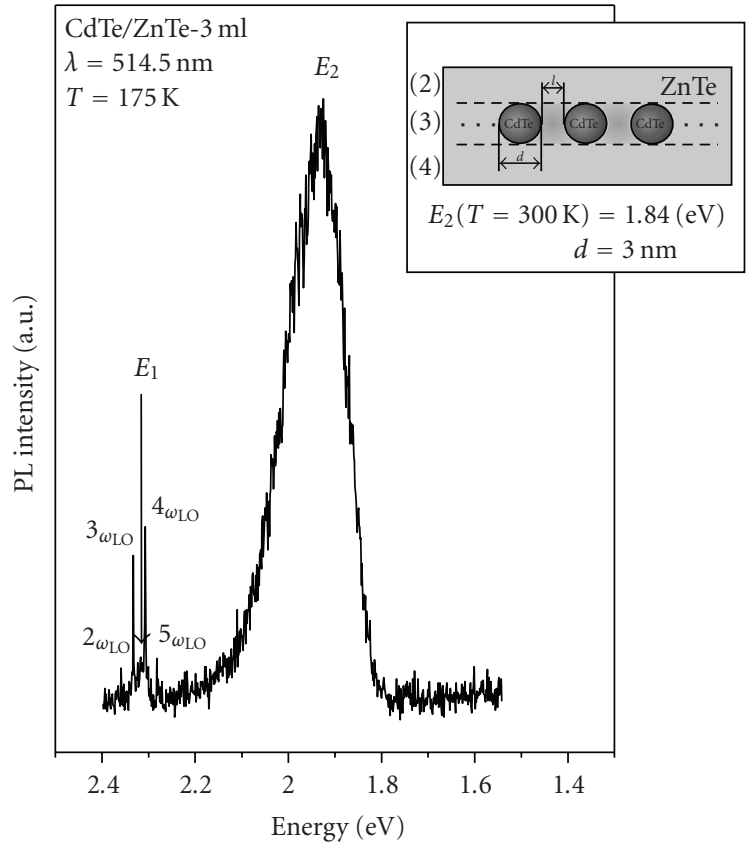

FIgURE 2: Typical PL spectra of sample with $3 \mathrm{ml}$ of CdTe in the finishing layer; PL spectra are excited by $\lambda=514.5 \mathrm{~nm}$ Ar-laser line and recorded at $175 \mathrm{~K}$. Insert: schematic presentation of the structure of SAQD.

$\mathrm{E}_{2}$ is about $20 \mathrm{meV}$. Red-shift of both bands with increasing temperature is below values one would expect from the temperature-induced shrinking of the ZnTe and CdTe band gap.

Band energies vary almost linearly with temperature, as presented in Figure 4 . The high-energy peak, $E_{1}$, is related to the band gap of the ZnTe barrier [9]. The low-frequency band, $E_{2}$, is related to the recombination in the CdTe quantum dots [5]. MP resonant processes are registered at temperature bellow $200 \mathrm{~K}$. This process is dominant for QDs with high emission energy, that is, presumably with smaller size [5].

Energies of localized states in QDs, experimentally registered by PL emission characteristics of the QDs, can be reasonably well described by the effective-mass approximation (EMA) with parabolic energy bands. Calculation of electronic transitions is done in EMA [10]. Parameters of CdTe and ZnTe, effective masses, and conduction and valence offsets are transferred from literature [11]. If we assume spherical symmetry of a particle, that is, that single sphere of CdTe is surrounded by ZnTe, calculated value of QD diameter is $3 \mathrm{~nm}$ in case of $3 \mathrm{ml}$ matches experimental $E_{2}$ value.

When adding a large quantity of CdTe between two layers of $\mathrm{ZnTe}$, it is expected to find larger QDs. As a consequence the energy $E_{2}$ would be lower. This expectation was not fulfilled for the $12 \mathrm{ml}$ sample; see Figure 4. To explain experimental results in $12 \mathrm{ml}$ case, we use model core/shell type nanostructures where QD is of gradient composition. CdTe core of radius $r_{C}$ is surrounded by concentric spherical 


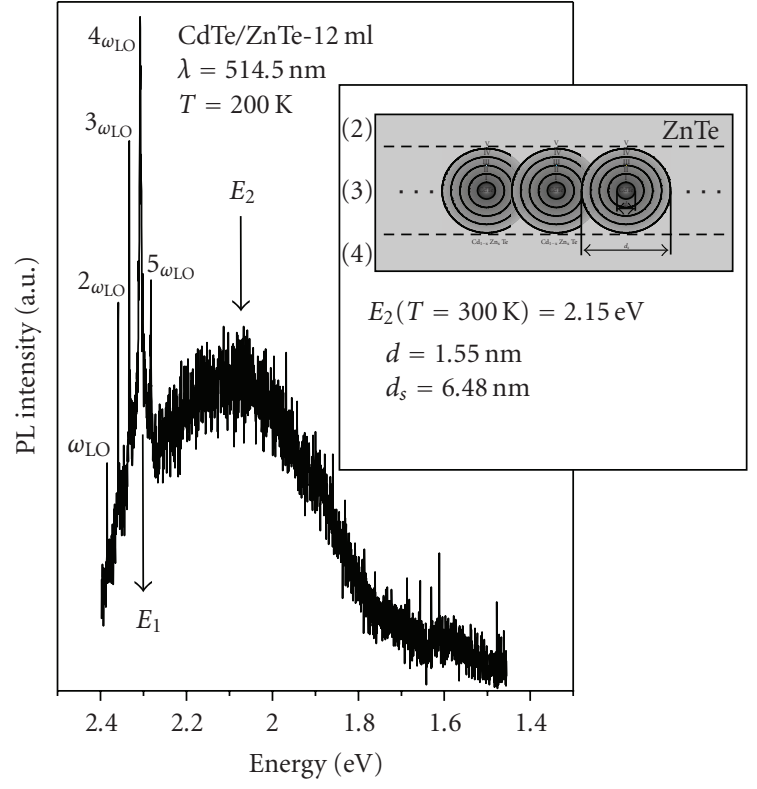

Figure 3: Typical PL spectra of sample with $12 \mathrm{ml}$ of CdTe in the finishing layer; PL spectra are excited by $\lambda=514.5 \mathrm{~nm}$ Ar-laser line and recorded at $200 \mathrm{~K}$. Insert: schematic presentation of the structure of SAQD.

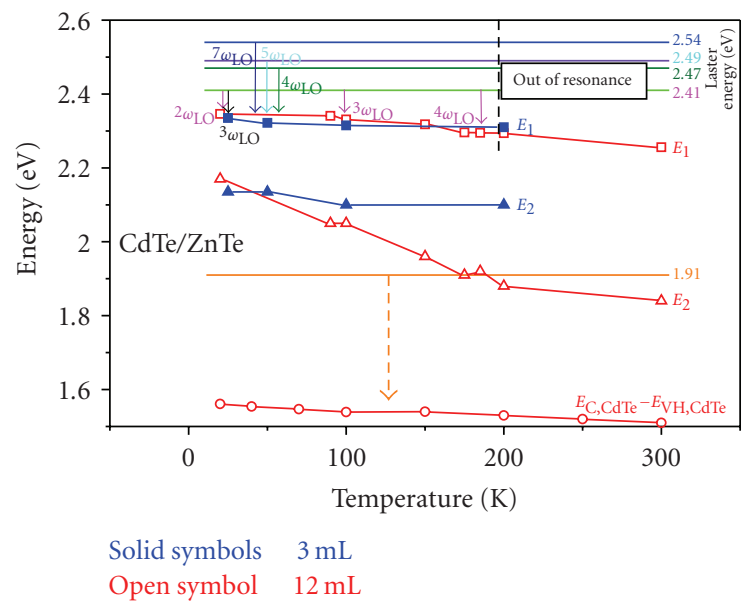

Figure 4: Temperature dependences of PL emission peaks and schematic presentation of multiphonon (MP) resonance. Results for $3 \mathrm{ml}$ sample are presented as open symbols, and for $12 \mathrm{~mL}$ sample as solid symbols.

layers each of $\mathrm{Cd}_{x} \mathrm{Zn}_{1-x}$ Te composition ( $x$ is proportional to the layer distance from the center) and finally from radius $r_{S}$ by $\mathrm{Cd}_{0.1} \mathrm{Zn}_{0.9} \mathrm{Te}$. We suppose that electrons and holes from QD are in spherical step-like energy well. Cd concentration in the surrounding $\mathrm{Cd}_{x} \mathrm{Zn}_{1-x} \mathrm{Te}$ gradually decreases to $10 \%$ $(x=0.1)$, step by step. We assumed that effective masses and conduction and valence offsets change proportionally to $x$. The existence of these intermediate layers, as a model of graded composition, influences rapidly electron, hole, and excitons spectra. For measured $E_{2}$ the diameter of the core is $1.55 \mathrm{~nm}$ and the diameter of outer sphere is $6.48 \mathrm{~nm}$.

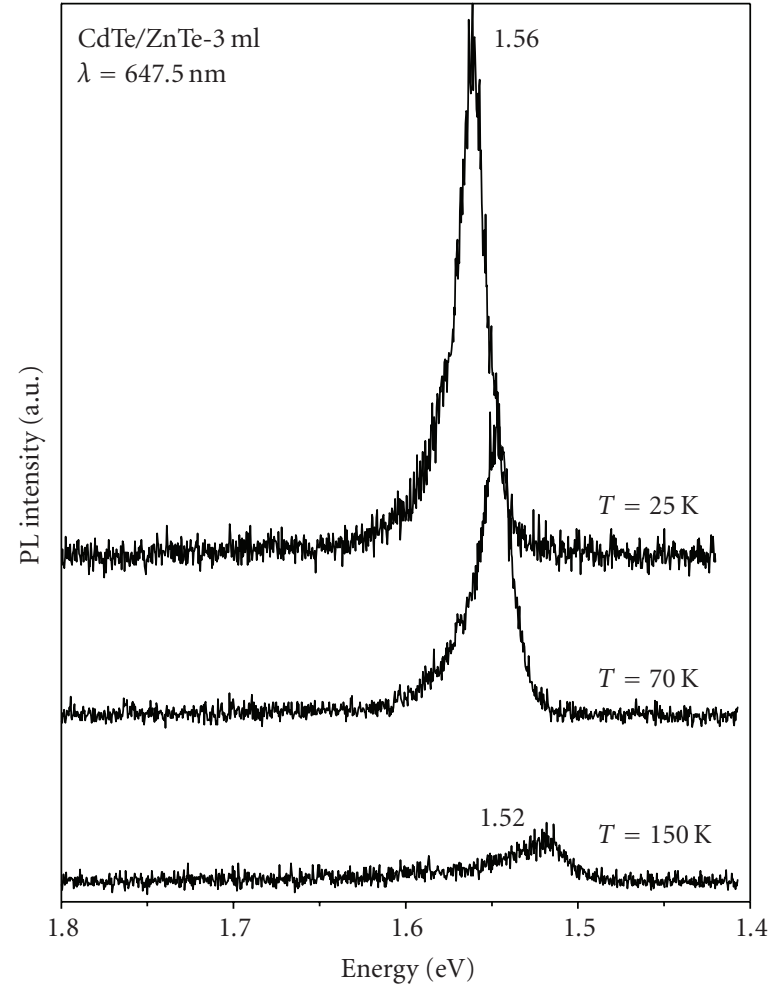

FIgure 5: Typical PL spectra of sample with $3 \mathrm{ml}$ of CdTe in the finishing layer; PL spectra are excited by $\lambda=647.5 \mathrm{~nm} \mathrm{Kr}$-laser line and recorded at different temperature.

In case of $3 \mathrm{ml}$ there is red-shift of the low-energy peak $\left(E_{2}\right)$ with increase of temperature, which is much larger than the shift one would expect from the temperature-induced shrinking of the CdTe band gap. In our opinion, even in the case of $3 \mathrm{ml}$, due to interdiffusion there are fluctuations of QD chemical composition and QD size distribution. So, we deal with the ensemble of QDs of different depths of the corresponding potentials. Thermally induced redistribution of carriers within the dot ensemble causes observed energy shift of photoluminescence energy. Opposed to that in the $12 \mathrm{ml} \mathrm{CdTe}$ sample all transitions are preserved due to relatively homogeneous thickness of the layer.

When the sample is excited by $\lambda=647.5 \mathrm{~nm} \mathrm{Kr}$-laser line, PL takes place only in the buffer layer of CdTe which is clearly seen in Figures 4 and 5 . The temperature dependences of PL emissions peaks and their intensities are identical to the dependences registered for the CdTe bulk crystal.

In principle, registered MP resonant process (see Figures 2 and 3.) can be described by the following equation: $E_{\text {in }}-$ $K \hbar \omega=E ; E_{\text {in }}$ and $E$ are the incident photon energy and electron transition energy, respectively [9], $\hbar \omega$ is the phonon energy, and $\mathrm{K}$ is the MP order.

MP emission is not registered at $T=300 \mathrm{~K}$. MP emission processes are registered at temperature below $T=200 \mathrm{~K}$ (for $2.41 \mathrm{eV}$ laser energy, $K=4$ ). The equation means that when the energy of the scattered photon approached the energy $E_{1}$, the Raman line becomes strongly enhanced. This is schematically presented by arrows on the top in Figure 4 . 


\section{Conclusion}

Structural and optical properties of $\mathrm{CdTe} / \mathrm{ZnTe}$ selfassembled quantum dots (SAQDs) growth by molecular beam epitaxy are investigated in this paper. Photoluminescence spectra consist of two main emission peaks: highenergy band connected to barrier band gap and low-energy band concerned to CdTe QD electron-hole recombination. Two mechanisms of relaxation are present in this system: relaxation directly to the CdTe quantum dot exciton ground state and optical phonon-assisted deexcitation. It seems that CdTe quantum dots when embeded in ZnTe barrier layers are very efficient carrier receivers. $\mathrm{CdTe} / \mathrm{Cd}_{x} \mathrm{Zn}_{1-x} \mathrm{Te}$ heterostructures inhomogenity modelled by gradual composition influence drastically basic transition energy. The present observations can help improve understanding of the optical and the microstructural properties in the CdTe/ZnTe self-assembled quantum dots. The registered multiphonon emission processes depend on temperature. When the energy of the scattered photon approached the energy $E_{1}$, the corresponding pon line becomes strongly enhanced.

\section{Acknowledgments}

This work was supported under the Agreement of Scientific Collaboration between the Polish Academy of Science and the Serbian Academy of Sciences and Arts. The work in Serbia was supported by the Serbian Ministry of Science (Project 141028).

\section{References}

[1] S. Mackowski, "CdTe/ZnTe quantum dots-growth and optical properties," Thin Solid Films, vol. 412, no. 1-2, pp. 96-106, 2002.

[2] S. Mackowski, G. Karczewski, T. Wojtowicz, et al., "Structural and optical evidence of island correlation in CdTe/ZnTe superlattices," Applied Physics Letters, vol. 78, no. 24, pp. 38843886, 2001.

[3] C. S. Kim, M. Kim, S. Lee, et al., "Evidence for 2D precursors and interdiffusion in the evolution of self-assembled CdSe quantum dots on ZnSe," Physical Review Letters, vol. 85, no. 5, pp. 1124-1127, 2000.

[4] H. Kirmse, R. Schneider, M. Rabe, W. Neumann, and F. Henneberger, "Transmission electron microscopy investigation of structural properties of self-assembled CdSe/ZnSe quantum dots," Applied Physics Letters, vol. 72, no. 11, pp. 1329-1331, 1998.

[5] T. A. Nguyen, S. Mackowski, H. E. Jackson, et al., "Resonant spectroscopy of 2-6 self-assembled quantum dots: excited states and exciton-longitudinal optical phonon coupling," Physical Review B, vol. 70, no. 12, Article ID 125306, 8 pages, 2004.

[6] N. Romčević, M. Romčević, R. Kostić, et al., "Far-infrared spectroscopy of CdTe/ZnTe self-assembled quantum dots," Journal of Alloys and Compounds, vol. 481, no. 1-2, pp. 6-9, 2009.

[7] N. Romčević, M. Romčević, R. Kostić, et al., "Resonant Raman spectra of CdTe/ZnTe self assembled quantum dots," Acta Physica Polonica A, vol. 116, no. 1, pp. 88-90, 2009.
[8] N. Romčević, M. Romčević, R. Kostić, D. Stojanović, G. Karczewski, and R. Galazka, "Raman spectra of CdTe/ZnTe self-assembled quantum dots," Microelectronics Journal, vol. 40, no. 4-5, pp. 830-831, 2009.

[9] S. L. Zhang, Y. T. Hou, M. Y. Shen, J. Li, and S. X. Yuan, "Multiphonon Raman scattering resonant with two kinds of excitons in a $(\mathrm{CdTe})_{2}(\mathrm{Znte})_{4} / \mathrm{ZnTe}$ short-period-superlattice multiple quantum well," Physical Review B, vol. 47, no. 19, pp. 12937-12940, 1993.

[10] M. Tkach, V. Holovatsky, O. Voitsekhivska, and M. Mikhalyova, "Exciton-photon interaction in the spherical nanoheterosystem CdS/B-," Physica Status Solidi B, vol. 203, no. 2, pp. 373-386, 1997.

[11] T. A. Nguyen, Investigation of electronic structure and optical properties of 2-6 self-assembled quantum dots, M.S. thesis, University of Cincinnati, Cincinnati, Ohio, USA, 2006. 


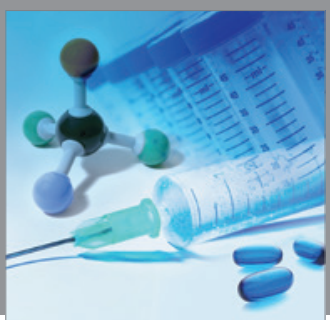

International Journal of

Medicinal Chemistry

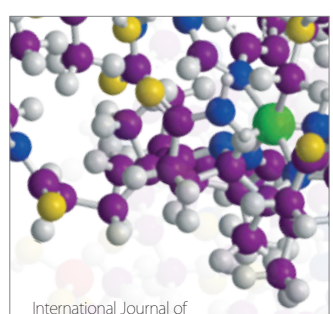

Carbohydrate Chemistry

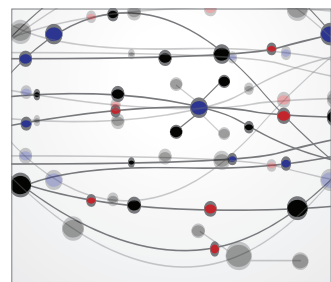

The Scientific World Journal
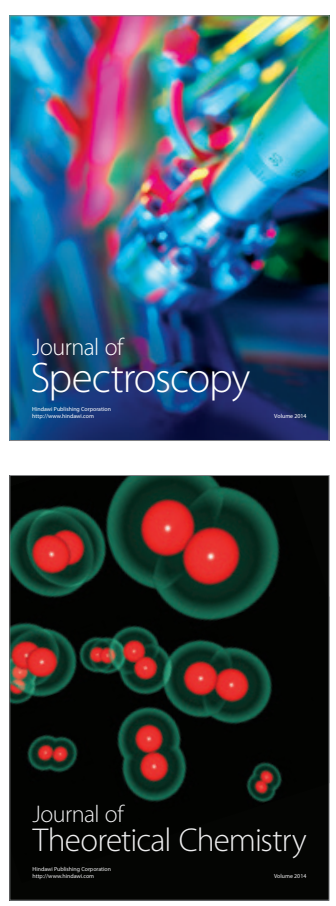
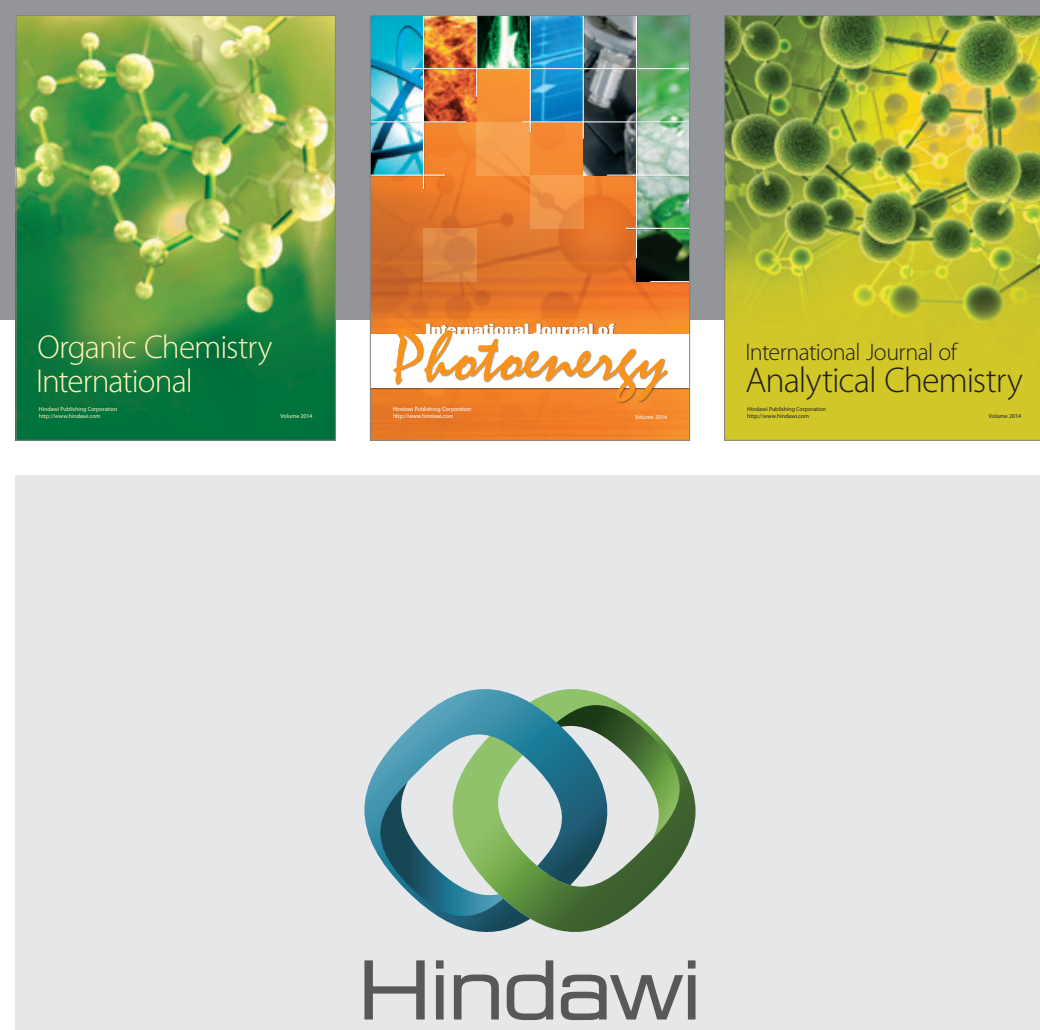

Submit your manuscripts at

http://www.hindawi.com
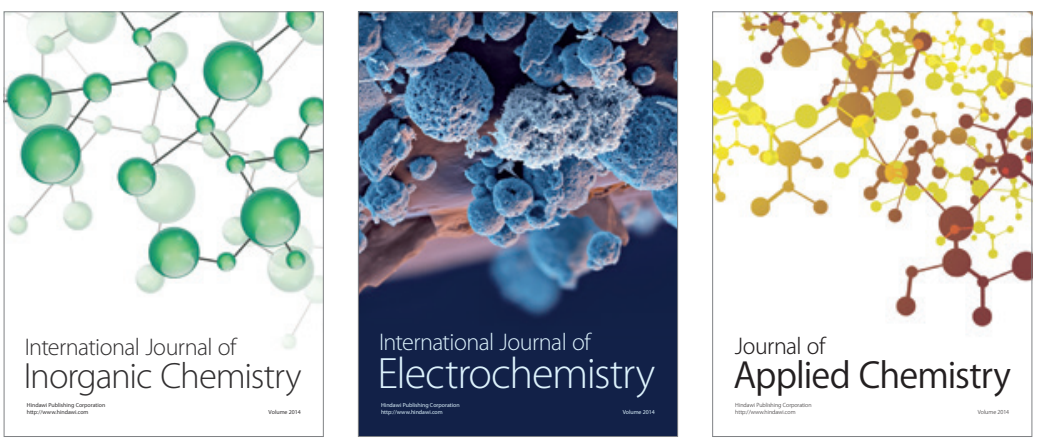

Journal of

Applied Chemistry
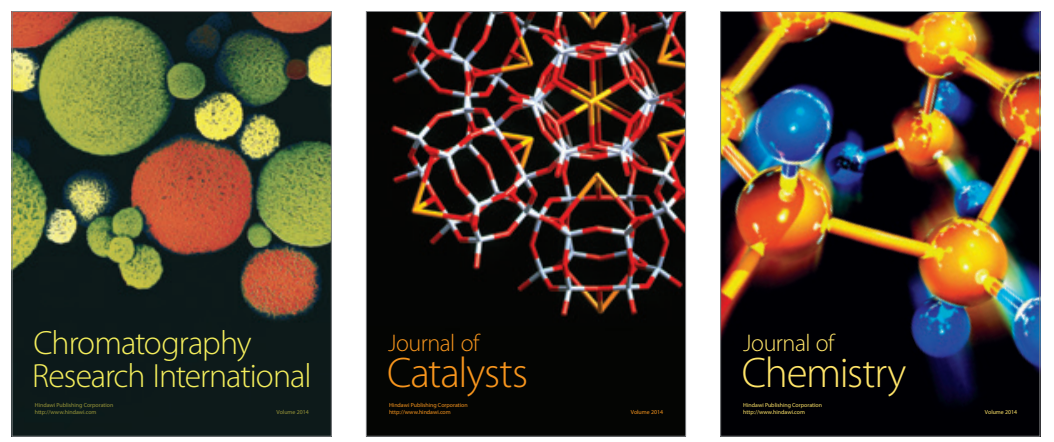
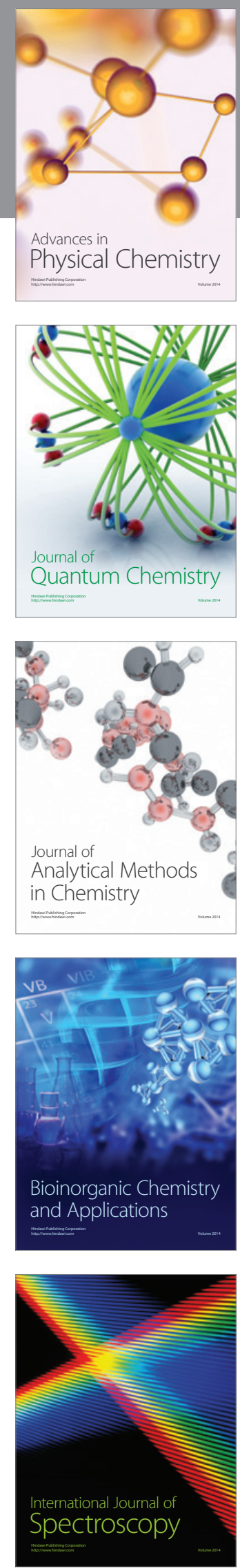